\title{
Regulation of Virulence Gene Expression in Bacteria: A Review
}

\author{
Dessie Abera \\ Animal Health Research Program, Livestock Research Process \\ Assosa Agricultural Research Center, Assosa, Ethiopia
}

\begin{abstract}
Bacterial diseases are the leading cause of death worldwide through the involvement of virulence determinants. Virulence genes encode virulence factors which are essential for pathogenesis and infectious disease development in bacteria. The virulence factor concept helps for further investigations in the fields of microbial pathogenesis. The objective of this paper is to review regulation of virulence gene expression in bacteria. Virulence genes can be acquired via spontaneous mutations or through horizontal gene transfer mechanisms. Bacteria sense and respond to environmental stimuli in the host during infection. Successful host infection can be possible by coordinated expression of multitude of virulence genes. They adapt in the host by modulating its gene expression. Pathogenic bacteria possess many regulatory networks that control the production of virulence factors which enable the bacteria to survive and proliferate in the host. The most common forms of regulation of genome expression are by involvement of transcription factors, post-transcriptional modifications, riboregulation, environmental flux sensing, stress response and biofilm formation through quorum sensing. Virulence gene expression is carefully controlled to ensure correct spatiotemporal production of virulence factors. Regulation of virulence gene expression is very complex and influenced by environmental factors. Major regulatory control mechanisms used by pathogenic bacteria are alternative sigma factors and the two component regulatory systems. They do not always express virulence factors because it is energetically costly and reduces bacterial fitness. Therefore, they need to regulate their virulence properly in order to maximize their fitness and it is a key invasion strategy of pathogens.
\end{abstract}

Keywords: Bacteria, Expression, Regulation, Virulence gene

DOI: $10.7176 / \mathrm{JBAH} / 11-3-02$

Publication date: February $28^{\text {th }} 2021$

\section{Introduction}

Bacteria monitor their extracellular physicochemical conditions and respond by modifying their genome expression (Ishihama, 2010). The bacterial pathogens within the host senses and adapts to the prevailing conditions by modulating its gene expression (Thomas and Wigneshweraraj, 2014). They regulate their virulence factors by the use of a number of common motifs (Wilson et al., 2002). Successful host infection depends on the coordinated expression of multitude of virulence factors of pathogenic bacteria and their genes involved in the infection process (Caldelari et al., 2013). The ability of a microbe to sense and respond to a myriad of environments during infection of the host determine its pathogenesis process. Virulence is a relative capacity of a microbe to cause damage in a host. The expression of the virulence gene of the microbe are regulated in response to environmental signals like temperature, availability of key metal ions, concentration of anions such as bicarbonate or phosphate, $\mathrm{pH}$, oxygen tension, and osmolarity encountered inside the infected host to initiate the appropriate and coordinate the adaptive response in gene expression (Winzer and Williams, 2001; Casadevall and Pirofski, 2009; Thomas and Wigneshweraraj, 2014).

Regulation of virulence gene is very complex and it is influenced by environmental factors in the host that can directly or indirectly affect gene expression. The most common forms of gene regulation are posttranscriptional modifications (e.g., methylation), an environmental sensing, multi-networks of regulation and biofilm formation can also be involved (Schroeder et al., 2017). Pathogenic bacteria possess intricate regulatory networks that temporally control the production of virulence factors, and enable the bacteria to survive and proliferate after host infections (Caldelari et al., 2013).

Virulence factor gene expression is carefully controlled in bacteria to ensure correct spatiotemporal production (Kröger et al., 2017). The expression of virulence gene is energetically costly for the organism and reduces bacterial fitness. So, pathogens need to regulate their virulence properly in order to maximize their fitness and a dynamic regulation of virulence factor expression is a key invasion strategy of pathogens (Kitamoto et al., 2016). Therefore, the objective of this review is to understand the virulence gene expression and their regulatory mechanisms in bacterial pathogens.

\section{Virulence genes in bacteria}

Virulence gene is every gene involved in pathogenicity or in causing disease (Wassenaar and Gaastra, 2001). For an individual bacterial pathogen, the total number of genes that can be categorized as virulence genes ranges from the low hundreds to more than one thousand depending upon the system under investigation (Thomas and 
Wigneshweraraj, 2014). Virulence genes encode virulence factors (Dirita et al., 2000). Bacterial virulence is a multifactorial process which are governed by a complex set of properties called virulence factors (Goebel and Hacker, 1989). The virulence factor concept has been a powerful engine in driving research and the intellectual flow in the fields of microbial pathogenesis and infectious disease study. The idea that pathogenic microbes are endowed with certain components that confer upon them the capacity for virulence is the central theme of virulence factor. Virulence is a relative quality and defined as the relative capacity of a microbe to cause damage in a host (Casadevall and Pirofski, 2009).

Virulence genes can be acquired via a variety of mechanisms, including spontaneous mutations and deliberate exchange of genes through horizontal gene transfer. Horizontal gene transfer allows the transmission of virulent genes both between individuals of a single species and between multiple species (Stewart and Costerton, 2001; Schroeder et al., 2017). Horizontal gene transfer is the incorporation of genetic elements which are blocks of DNA containing mobile genetic elements. Those may contain large blocks of virulence determinants (adhesins, invasions, toxins, protein secretion systems, or antibiotic resistance mechanisms). It is a rapid, allowing dramatic change of the genetic makeup of bacteria which plays a principal part in the molecular evolution of novel bacterial pathogens (Wilson et al., 2002).

Bacteria sense and respond to environmental stimuli during infection of the host through the use of a repertoire of genetic functions which are independently regulated in response to environmental signals encountered inside the infected host (Wilson et al., 2002). The microbial attributes that confer the potential for virulence fall within several categories: the ability to enter a host; the ability to evade host defenses; the ability to grow in a host environment; the ability to counteract host immune responses; the ability to acquire iron and nutrients from the environment and the ability to sense environmental changes (Casadevall and Pirofski, 2009). The products of such genes that facilitate the successful colonization and survival of the bacterium to cause damage to the host are considered as virulence or pathogenicity determinants (Thomas and Wigneshweraraj, 2014). Infectious diseases through virulence determinants are the leading cause of death (Wilson et al., 2002).

\section{Regulation of virulence gene expression}

Regulation of virulence gene expression is crucial for pathogenic bacteria (Peggy and Victor, 2000). Bacterial gene expression is carefully controlled (Kröger et al., 2017). Pathogenic bacteria possess intricate regulatory networks that temporally control the production of virulence factors, which enable the bacteria to survive and proliferate after host infections. It is a key invasion strategy of pathogens (Caldelari et al., 2013; Kitamoto et al., 2016).

\subsection{Types of regulation}

Transcription initiation is the major step of genome expression regulation in bacteria. mRNA abundance is also regulated at the step of elongation and termination, and its degradation is subject to control (Ishihama, 2010). In addition, post-transcriptional regulation plays a major part in coordinating gene expression networks (Romeo et al., 2013).

\subsubsection{Transcription factors}

Transcription factors determine the pattern of genome transcription through control of a limited number of RNA polymerase distribution. These factors are composed of two domains, one functioning as the sensor for external and internal signals and the other as interacting DNA targets. One group of transcription factors, known as negative regulators or repressors, acts by binding to target operators in the absence of low-molecular weight effectors, known as inducers. Promoters under the control of repressors are inactive, but become active once the repressors are dissociated from target DNA after association with the inducers. In contrast, another group of transcription factors, known as positive regulators or activators, require interaction with effector ligands to function (Ishihama, 2010).

Transcription factors can be classified into specific (local) and global regulators. Global regulators are a small number of transcription factors, which influences the expression of a large number of transcription units that belong to different metabolic pathways, thereby exhibiting pleiotropic phenotypes (Pérez-rueda and Collado-vides, 2000; Janga et al., 2007). Usually transcription factors can regulate gene expression directly or indirectly through global gene regulators for virulence (Schroeder et al., 2017). On the other hand, specific (local) regulators are a large number of transcription factors, which affects the expression of one specific gene or a small number of transcription units (Marti'nez-Antonio and Collado-vides, 2003; Wei et al., 2004). The role of both specific and global regulators is to mediate precise activation or repression by sensing changes in environmental conditions or metabolic states. A single putative global regulator is capable of regulating genes transcribed by different $\sigma$ factors and it is subject to specific transcription factors (TFs) regulation (Marti'nezAntonio and Collado-vides, 2003).

Regulation of virulence genes where the transcription factors bind directly to the promoter region has been seen in Enterohemorrhagic Escherichia coli (EHEC) where the transcription factors Cra, KdpE, and FusR bind 
directly to the ler region on the locus of enterocyte effacement (LEE) pathogenicity island in order to control the gene expression (Mohanty and Kushner, 2006).

\subsubsection{Post-transcriptional regulations}

The multifactor promoters and the multi target transcription factors can be assembled into complex regulatory networks of transcription that play important roles in the coordination of bacterial metabolism and physiology in response to the environment (Ishihama, 2010). In addition, post-transcriptional regulation plays a major part in coordinating gene expression networks (Romeo et al., 2013). Post-transcriptional modifications act as a quality control mechanism to produce stable RNA transcripts, which occur much more commonly in prokaryotes (Mohanty and Kushner, 2006).

Two kinds of post-transcriptional systems predominate in many proteobacteria: trans-acting RNAs that use an RNA chaperone protein which promotes the interaction of numerous base-pairing small RNAs (sRNAs) to pair with mRNA targets (Gottesman and Storz, 2011). And, the other one is Csr (carbon storage regulator) or Rsm (repressor of stationary-phase metabolites) systems, which exploit a sequence-specific RNA-binding protein, referred to as CsrA, RsmA or RsmE to alter the translation and/or stability of mRNA targets that regulates gene expression post-transcriptionally by affecting ribosome binding and/or mRNA stability. CsrAmediated repression typically involves CsrA binding to multiple sites in the untranslated leader and/or initially translated region of target transcripts (Babitzke and Romeo, 2007). Thus, bound CsrA represses translation by competing with ribosome binding (Baker et al., 2002; Dubey et al., 2003), which leads to rapid degradation of the polycistronic transcript (Romeo et al., 2013). Polyadenylation of RNAs by poly (A) polymerase I (PAP I) in Escherichia coli plays a significant role in mRNA decay and general RNA quality control. However, many important features of this system, including the prevalence of polyadenylated mRNAs in the bacterium, are still poorly understood (Mohanty and Kushner, 2006).

Post-translational modifications are a well-recognized mode of regulations (Schroeder et al., 2017). In Salmonella enterica the translation of the virulence associated effector protein, AvrA which was completely abolished, while transcription was unaffected when the genes CsrA/CsrB were deleted. The expression of AvrA is regulated by a post transcriptional modification induced by the effective concentration of CsrA, which is achieved by sequestering of CsrB (Kerrinnes et al., 2009). Other post transcriptional regulators also participate in bacterial virulence networks, including RNA helicases which unwind their RNA substrates in an ATPdependent reaction, and are central to all cellular processes involving RNA (Steimer and Klostermeier, 2012); and the Crc protein which is post-transcriptional regulator of Pseudomonas, those proteins are a global regulator that controls the metabolism of carbon sources and catabolite repression in Pseudomonas, which determine its contribution to the intrinsic antibiotic resistance and virulence of $P$. aeruginosa (Linares et al., 2010; Moreno et al., 2014; Reales-calderón et al., 2015).

One study showed Crc deficient mutants of $P$. aeruginosa were found to secrete lower levels of virulence factors, including toxins and proteins necessary for cytolysis; which is hypothesized to be the reason the mutant was also had reduced cytotoxicity in comparison to the wild type strain. Lowered amounts of ToxA, virulence determinant exotoxin A, were also found in the vesicle-free secretome of $P$. aeruginosa crc deficient mutant when compared to the wild type (Reales-calderón et al., 2015). These, all factors influence gene expression and virulence (Vakulskas et al., 2015).

\subsubsection{Ribo-regulation}

RNA binding elements are responsible for post-transcriptional modifications, but RNA species are also responsible for ribo-regulation of virulence genes (Dar et al., 2016). Cis-regulation of RNA elements are known as riboswitches or attenuators, which are often present in the gene's 5 ' untranslated region (5' UTR). It can cause early termination of transcription by direct ligand binding in order to control bacterial gene expression. In the presence of different metabolite concentrations, the riboswitch structure is altered upon ligand binding, which allows the full expression of the gene due to destabilization of the terminator (Bjarnsholt et al., 2010; Dar et al., 2016). L. monocytogenes, for example has 55 recognized riboswitches that respond to 13 distinct ligands to control virulence (Schroeder et al., 2017). Riboswitches impact virulence and metabolism (Oliva et al., 2015). They are essential to gene regulation involved in bacterial physiology and virulence, and may provide potential targets for new drug therapies (Schroeder et al., 2017).

Noncoding regulatory RNA molecules (ncRNAs), also known as small RNAs (sRNAs), participate in several of these global regulatory networks (Storz et al., 2005). Small RNA regulators (sRNAs) have been identified in a wide range of bacteria and found to play critical regulatory roles in many processes (Gottesman and Storz, 2011). The importance of small, noncoding RNAs that act as regulators of transcription for RNA modification or stability, and of mRNA translation is becoming increasingly apparent. Many of the regulatory RNAs act through base-pairing interactions with target RNAs and other regulatory RNAs act by modifying protein activity, in some cases by mimicking the structures of other RNA or DNA molecules (Storz et al., 2005).

\subsubsection{Environmental flux sensing and response to changes}

A central principle in effective regulation of virulence is the ability to sense and respond to the bacteria's 
external environment (Schroeder et al., 2017). Bacterial cells constantly monitor extracellular physicochemical conditions, so that they can respond by modifying their gene expression patterns to adjust growth (Rui and TseDinh, 2003). The link between changes in environmental conditions and changes in transcriptional regulation involves signal-transduction pathways, which may involve the direct production of an isomer such as allolactose (Marti'nez-Antonio and Collado-vides, 2003), or a cascade of enzymatic modifications such as the activation of the nitrogen regulator in response to nitrogen limitation (Reitzer, 2003). The precise link with regulatory proteins is achieved by small metabolites (i.e. products of active metabolism (Martı'nez-Antonio and Collado-vides, 2003).

\subsubsection{Stress response}

Multiple stress response pathways have been identified as key players in the regulation of virulence (Schroeder et al., 2017). Environmental factors, such as oxygen concentration can impact the effect of transcription factors on virulence genes. It was shown that Cra strongly activates expression of the locus of enterocyte effacement (LEE) pathogenicity island under aerobic conditions, while under these same conditions FusR represses expression (Grant et al., 2003).

\subsubsection{Complex multi-regulation networks}

Virulence gene regulation is complex and a mutation in the typA gene in $P$. aeruginosa lowered virulence, thereby evading phagocytosis, increasing attachment, and enhancing biofilm formation. Additionally, this mutation, which initially may be viewed as a fitness cost led to down regulation of the type III secretion system (Neidig et al., 2013).

\subsubsection{Biofilm formation through quorum sensing}

Bacteria that adhere to implanted medical devices or damaged tissue can encase themselves in a hydrated matrix of polysaccharide and protein, and form a slimy layer known as a biofilm (Stewart and Costerton, 2001). Biofilm formation is an essential part of pathogenicity (or a combination of virulence and antibiotic resistance); thus, the formation is highly regulated by a complex network of transcriptional regulation (Davey and Toole, 2000; Sauer et al., 2002). The initiation of biofilm formation not only begins with quorum sensing, which may be considered a virulence regulator, but the resistance of the biofilm structure itself may also be considered a regulator of virulence. Once a biofilm is established, it can activate additional virulence genes within the biofilm again through quorum sensing or two-component systems (Schroeder et al., 2017). Biofilms usually are made up of multiple species of bacteria, which are more difficult to treat and caused more persistent infections (Lee et al., 2013), and it is clear that the establishment of a biofilm enhances virulence (Schroeder et al., 2017).

Biofilm formation facilitate communication amongst bacteria and for regulation of a variety of other virulence factors (Lee et al., 2013). Bacteria communicate either through quorum sensing or two component systems, which are directed and deliberate forms of communication of bacteria (Schroeder et al., 2017). It also facilitate the horizontal transfer of virulence genes among individuals of a single species or between multiple species. Of course, such a transfer is facilitated by proximity; thus, when bacterium convert from planktonic, free floating individuals, to a sessile collective, transfer via plasmids is hastened (Stewart and Costerton, 2001; Schroeder et al., 2017). Horizontal gene transfer is a rapid and dramatic change of the genetic makeup of bacteria. Recent evidence has shown that horizontal gene transfer plays a principal part in the molecular evolution of novel bacterial pathogens (Wilson et al., 2002). Horizontally acquired genes are common to E. coli, which contribute to the disease process, and increased virulence gene expression through coordinated expression of Enterohemorrhagic Escherichia coli virulence genes. These enables the bacterium to cause hemorrhagic colitis and the complication known as hemolytic-uremic syndrome (Mellies and Lorenzen, 2014).

\subsection{Regulatory control mechanisms}

The genetic control of virulence can be divided into two broad categories: random and nonrandom. Random forms of control include cases in which a fraction of a given population exhibits a phenotype different from the rest of the population. Such phenomena are usually referred to as phase or antigenic variation and typically involve surface structures such as flagella, pili, outer membrane proteins, and capsules that enhance the colonization of host tissues or allow the microbe to evade phagocytosis. The second class of genetic control is nonrandom. Bacteria sense signals in the environment and respond accordingly by expressing gene products necessary for survival in the host (Dirita and Mekalanos, 1989). But, based on major regulatory control mechanisms used by pathogenic bacteria to control expression of virulent genes, it can be classified into alternative sigma factors and the two component regulatory systems (Wilson et al., 2002).

\subsubsection{Sigma factors regulatory system}

Sigma factors are protein subunits of bacterial RNA polymerases and control the initiation of transcription at the promoter sequence. They are a major regulator of prokaryotic gene expression. Bacteria use different sigma factors to control the initiation of different promoters including those promoters whose genes encode virulence factors. In particular, the alternative sigma factors RpoS $(\sigma 38)$ has been shown to regulate the expression of genes in response to stationary phase, nutrient deprivation, and oxidative and osmotic stress. The RpoS sigma 
factor has been shown to be important for virulence in a number of bacterial pathogens, including Salmonella typhimurium, E. coli, P. aeruginosa, and L. pneumophila (Dirita and Mekalanos, 1989; Wilson et al., 2002).

\subsubsection{Two component regulatory systems}

Two-component systems (TCS) are ubiquitous systems of signal transduction in bacteria (Kröger et al., 2017). It allow bacteria to adapt to environmental changes in response to various cues such as nutrient concentrations, cell population density, antibiotics, ionic strength and membrane disturbances (Gordon et al., 2013). Virulence can be enhanced via two-component bacterial communication systems (Schroeder et al., 2017). It consists of two key proteins involved in the expression of virulence determinants. These are, a sensor protein usually a bacterial membrane-associated histidine kinase, which senses different physiological conditions of the bacterial cell and a response regulator which usually binds to the promoter region of a gene to activate or repress transcription (Wilson et al., 2002; Gordon et al., 2013; Schroeder et al., 2017).

Large scale alterations in the transcriptional profile of virulence genes in the bacterium occur in response to environmental signals (Thomas and Wigneshweraraj, 2014). And, two component regulatory systems are involved in environmental factors like iron, phosphate, nitrogen, carbon, capsule production, temperature and flagellar activity (Wilson et al., 2002). Temperature-dependent Bvg system of the Bordetellae and the yop genes of Yersinia species that are activated upon entry into mammalian or human hosts, or the role of the Fur repressor in regulating iron acquisition systems (Thomas and Wigneshweraraj, 2014). Bacterial virulence factors known to be regulated by two component systems include pili formation and cholera toxin production of $V$. cholerae (ToxR/ToxS64), Salmonella survival in macrophages (PhoP/PhoQ65), outer membrane porin regulation in Salmonella and E. coli, and iron regulation in Salmonella and Pseudomonas (Wilson et al., 2002).

Two-component system in $S$. aureus that control multiple cellular function, is essential to virulence and is being investigated as a potential target to combat Staphylococcus infections (Schroeder et al., 2017). In pathogenic bacterium, Staphylococcus aureus, the SaeRS two-component system (TCS) plays a major role in controlling the production of over 20 virulence factors including hemolysins, leukocidins, superantigens, surface proteins and proteases. The SaeRS TCS is composed of the sensor histidine kinase SaeS, response regulator SaeR, and two auxiliary proteins SaeP and SaeQ (Cheung et al., 2004; Liu et al., 2016).

\subsection{Environmental cues regulate virulence factor expression}

Bacteria use a wide variety of mechanisms for sensing and responding to environmental changes (Babitzke and Romeo, 2007). Pathogens do not always express virulence factors because of the associated fitness cost. It has been reported that mutant strains deficient in virulence factors display increased fitness compared to their virulent counterparts, due to the cost of virulence. Therefore, it is ideal for pathogens to stay avirulent before reaching their colonization sites and only to express virulence factors upon reaching the destination sites (Kitamoto et al., 2016). The regulation and determination of time of expression of virulence factors for most pathogenic bacteria at the right time and location is suggested with evidence that pathogens are able to sense the microenvironment in the host (Wilson et al., 2002; Kitamoto et al., 2016). Most pathogens will respond to various environmental cues to regulate their virulence gene expression (Kitamoto et al., 2016).

The ability of microorganisms to respond rapidly to changes in the environment is crucial for their survival. Bacteria have developed several regulatory strategies that ensure their survival during the transition from exponential growth into stationary phase as a result of nutrient starvation. During this transition, the carbon storage regulatory system (Csr) of Escherichia coli plays an important role in controlling gene expression. The effector of Csr is a small RNA-binding protein, CsrA (carbon storage regulator A). Previous studies have established that CsrA represses gluconeogenesis, glycogen biosynthesis and catabolism; and activates glycolysis, cell motility and acetate metabolism (Baker et al., 2002; Dubey et al., 2003). In addition, CsrA regulates biofilm formation. Biofilms often complicate chronic and difficult-to-treat infections by protecting bacteria from the immune system, decreasing antibiotic efficacy, and dispersing planktonic cells to distant body sites (Jackson et al., 2002).

Environmental cues sensed by two-component signal transduction systems and other regulatory factors govern the expression of the CsrA-binding sRNAs and, ultimately, the effects of CsrA on secretion systems, surface molecules and biofilm formation, quorum sensing, motility, pigmentation, siderophore production and phagocytic avoidance (Babitzke and Romeo, 2007; Vakulskas et al., 2015).

\subsubsection{Physico-chemical sensing}

Enteric pathogen for example sense their microenvironment for bicarbonate, $\mathrm{pH}$, oxygen tension and osmolarity which helps for their successful intestinal colonization. Certain pathogens, such as Enteropathogenic E. coli (EPEC), express GadX in response to acid stimulation, an activator of genes involved in acid tolerance. GadX is a member of transcriptional regulators and is a positive regulator of acid tolerance genes and a suppressor of virulence genes, which are not required in the acidic environment (Kitamoto et al., 2016). Enterohemorrhagic $E$. coli (EHEC) survive in the acidic environment of the stomach through different acid resistance systems, like a glucose-repressed or oxidative system, and glutamate and arginine-dependent systems (Mellies and Lorenzen, 
2014).

The osmolarity of the intestinal lumen is known to be higher than that of the tissue. Certain pathogens express the transmembrane histidine kinase EnvZ, a sensor of environmental osmolality. During conditions of high osmolality, EnvZ auto-phosphorylates and transfers the phosphoryl group to the response regulator OmpR, resulting in the formation of phosphorylated OmpR and the pathogen expresses flagellin but in response to a relative decrease in osmolarity, EnvZ/OmpR induce the expression of tviA gene which represses the biosynthesis of flagellin, and enhances capsule production through the activation of the viaB locus. This reciprocal expression of virulence related genes is thought to be of crucial importance to the pathogen as it allows it to evade innate immune system at the intestinal mucosa and contributes to the increased systemic dissemination of pathogens (Kitamoto et al., 2016).

Adaptive responses of bacteria to low oxygen environments in the presence of reactive oxygen species (ROS) are key to the survival of several pathogens in the host. Hypoxia or anoxia is used as a signal not only to trigger changes in expression of genes that allow adaptation to the lack of oxygen but also upregulate the activity of genes that result in obvious damaging consequences for the cell by encoding toxins (Thomas and Wigneshweraraj, 2014). During S. typhimurium infection, the anaerobic response regulator and nitrate reductase (FNR) is activated in response to oxygen and enhances the expression of several loci related to flagellar biosynthesis, chemotaxis, anaerobic carbon utilization and the Salmonella pathogenicity island (SPI)-1 genes. The activation of FNR leads to elongation of type 3 secretion system (T3SS) needles. FNR is known to be widely conserved among enteric pathogens, suggesting the importance of oxygen in the regulation of virulence (Kitamoto et al., 2016).

In bicarbonate-rich host environment, several pathogens, such as Streptococcus pyogenes and EHEC, have evolved a bicarbonate associated signal transduction system to control the virulence factors involved in bacterial colonization of the host surfaces (Kitamoto et al., 2016).

\subsubsection{Sensing of metabolites}

Bile has potent antimicrobial properties and it has an important role in host defense. It is evident that certain pathogens are able to tolerate it and use it to their advantage (Kitamoto et al., 2016). In the pathogenesis of $V$. cholerae infection, the major virulence factors of $V$. cholera are cholera toxin (CT, encoded as ctxAB) and toxincoregulated pilus (TCP, encoded as tcpA), both positively regulated by the dimerized transcription activator ToxT (Dirita and Mekalanos, 1989). In vivo, bile has a severe down-modulating effect on activation of cholera toxin and pilus expression (Dirita et al., 2000). Unsaturated fatty acids present in bile directly bind ToxT, preventing its homo-dimerization and repressing ctxAB and tcpA genes. But, the expression of flagellar genes is enhanced which enables it to penetrate the thick mucus layer and adhere to the underlying epithelial cells where bile concentration is low, CT and TCP activated. Since TCP expression carries a fitness cost and CT-induced fluid secretions from epithelial cells could flush away the pathogen from the cellular surface, the bile-mediated regulatory mechanism allows the pathogen to successfully approach the epithelial barrier (Kitamoto et al., 2016).

E. coli expressing type 1 pili (fimbriae) adhere to mannose containing molecules on many types of eukaryotic cells. Adherent Fim + bacteria compete better for essential growth nutrients than do Fim - strains and a Fim + strain that elicits the $E$. coli heat labile toxin is more toxic than a Fim - strain. This type 1 producing $E$. coli alternates between the Fim + and Fim - states and this phase variation is under transcriptional control since it is very important for pathogenicity (Dirita and Mekalanos, 1989).

Some pathogens are capable of sensing Short-chain fatty acids (SCFAs). This sensory ability is often connected to regulation of virulence gene expression. For example, S. typhimurium uses SCFA concentration and composition to regulate its invasion genes encoded on the Salmonella pathogenicity island 1 (SPI-1) via the BarA/SirA two-component regulatory system (Kitamoto et al., 2016).

\subsubsection{Temperature sensing}

In pathogenic bacteria virulence gene expression is modulated by environmental parameters and temperature is a key among the factors. Transcription of virulence genes in bacteria infecting warm-blooded hosts are induced upon a shift from low environmental to a higher host temperature (Guijarro et al., 2015). Thus, bacteria have developed precise and defined regulation systems to modulate the expression of specific genes in response to temperature shifts (Steinmann and Dersch, 2013). Different molecular mechanisms are involved in sensing temperature changes in mammal-pathogenic bacteria. Most of them are related to changes in DNA, RNA or protein conformation and alteration in membrane structure (rigidification) which determines in some cases the activation of a two-component signal transduction pathway (Steinmann and Dersch, 2013; Guijarro et al., 2015). In vivo, a temperature of $37^{\circ} \mathrm{C}$ has a severe down-modulating effect on activation of cholera toxin and pilus expression (Dirita et al., 2000).

Shigella virulence is strictly regulated by temperature. This effect is at the level of gene expression and the product of the virR gene activate expression of plasmid encoded loci at high temperature (Dirita and Mekalanos, 1989). 


\subsubsection{Sensing of mechanical stimuli}

Pathogen attachment to host cells is a crucial step in the development of infection and attachment triggers expression of GrlA which is a cytoplasmic regulator of virulence genes (Kitamoto et al., 2016). Bacterial pathogens use perturbations or damage of their membrane(s) induced by certain offensive external stimuli which interpreted as a signal that they have encountered hostile elements of the host immune system and then it modulate the expression of more overt virulence functions like protein secretion (Thomas and Wigneshweraraj, 2014).

\subsubsection{Quorum sensing}

Bacteria have the ability to regulate gene expression in response to population density by a process known as quorum sensing (Romeo et al., 2013). Quorum sensing regulation is a cell-cell communication which involves the direct or indirect activation of a response regulator by a small diffusible signal molecule (Winzer and Williams, 2001). The bacterial cell-to-cell communication is mediated by the production of signaling molecules, called autoinducers (AI) (Eberl, 2006; Schroeder et al., 2017). The regulation and timing of expression of virulence factors is very important for most pathogenic bacteria (Wilson et al., 2002). As the bacterial population increases those small signal molecules accumulate outside the cell (in the environment) and bacteria monitor this information, once their concentration reaches a critical value (the threshold), specific receptors mediate the induction or repression of target gene expression to track changes in bacterial density (Kitamoto et al., 2016; Sousa et al., 2017). The coupling of virulence factor production with high bacterial population density ensures that the mammalian host lacks sufficient time to mount an effective defence against consolidated attack. Such a strategy depends on the ability of an individual bacterial cell to sense other members of the same species and in response, differentially express specific sets of genes (Winzer and Williams, 2001).

Global changes in virulence gene expression can also be implemented in response to fluctuations in a single environmental parameter and can involve a single regulatory mechanism with some quorum sensing regulon (Thomas and Wigneshweraraj, 2014). A common quorum sensing system in Gram-negative bacterial species involves the synthesis and detection of N-acyl-L-homoserine lactones (AHLs). Since AHLs can freely diffuse across cell membranes, as the bacterial population increases, the concentration of AHLs in the cellular environment increases. Once a critical level of AHL is reached, AHL re-enters the cell and is detected by a response regulator, thereby altering expression of target genes (Romeo et al., 2013). Post-translational modified peptides of quorum-sensing signal molecules play a great role in Gram-positive bacteria as AHLs is for Gramnegative bacteria. For example, Pseudomonas aeruginosa employ AHLs and Staphylococcus aureus employ peptides to control the expression of multiple virulence genes in concert with cell population density. And others like N-(3-oxododecanoyl) homoserine lactone, possess intrinsic pharmacological and immunomodulatory activities such that they function as virulence determinants. The mutation of genes involved in either quorumsensing signal generation or signal transduction frequently results in the attenuation of virulence (Winzer and Williams, 2001).

Quorum sensing negatively influences virulence gene expression in certain toxigenic Vibrio cholerae strains (Kovacikova and Skorupski, 2002). At low cell density, when the concentrations of autoinducers are low, the response regulator LuxO is phosphorylated by a relay from the sensor proteins. This activated form of LuxO results in reduced expression of hapR, thus permitting high-level expression of the virulence cascade. At high cell density, binding of auto inducers to their cognate sensors leads to dephosphorylation of LuxO. LuxO no longer reduces hapR expression, and HapR, in turn, functions to down regulate expression of the virulence cascade by reducing expression from the tcpPH promoter (Miller et al., 2002; Zhu et al., 2002). In addition to quorum-sensing regulation, $V$. cholerae virulence factors, cholera toxin and the toxin-coregulated pilus production are strongly influenced by environmental conditions. The well-characterized ToxR signal transduction cascade is responsible for sensing and integrating the environmental information and controlling the virulence regulon (Zhu et al., 2002).

\section{Conclusions}

Virulence determinants of bacterial pathogens are essential for bacterial infectious disease development. Virulence gene is a gene involved in pathogenicity. They can be acquired via a variety of mechanisms, including spontaneous mutations or through horizontal gene transfer mechanisms. Expressions of virulence genes in bacteria is regulated carefully to produce at the right time and location because the expression of virulence genes is energetically costly for the organism and properly controlled in order to maximize their fitness. The regulation of virulence gene expression is very complex and regulation of these genes is influenced by environmental factors. Bacteria constantly monitor their environment through a wide variety of mechanisms so that they can sense, respond and adapt by modulating their genome expression to environmental changes. The ability of bacterial pathogens to respond rapidly to changes in the environment is crucial for their survival. Major regulatory control mechanisms used by pathogenic bacteria to control expression of virulent genes are alternative sigma factors and the two component regulatory systems. Proper regulation of virulence factor expression is very 
important to maximize their fitness and it is a key invasion strategy of bacterial pathogens.

\section{References}

Babitzke P, Romeo T (2007). CsrB sRNA family: sequestration of RNA-binding regulatory proteins. Current Opinion in Microbiology. 10: 156-163. https://doi.org/10.1016/j.mib.2007.03.007

Baker CS, Morozov I, Suzuki K, Romeo T, Babitzke P (2002). CsrA regulates glycogen biosynthesis by preventing translation of glgC in Escherichia coli. Molecular Microbiology. 44: 1599-1610.

Bjarnsholt T, Tolker-nielsen T, Høiby N, Givskov M (2010). Interference of Pseudomonas aeruginosa signalling and biofilm formation for infection control. Expert Reveiws in Molecular Medicine. 12: 1-19. https://doi.org/10.1017/S1462399410001420

Caldelari I, Chao Y, Romby P, Vogel J (2013). RNA-Mediated Regulation in Pathogenic Bacteria. Cold Spring Harbor Perspectives in Medicine. 3: 1-22.

Casadevall A, Pirofski L (2009). Virulence factors and their mechanisms of action: the view from a damageresponse framework. Journal of Water and Health. 07(S1): 2-18. https://doi.org/10.2166/wh.2009.036

Cheung AL, Bayer AS, Zhang G, Gresham H, Xiong YQ (2004). Regulation of virulence determinants in vitro and in vivo in Staphylococcus aureus. FEMS Immunology and Medical Microbiology. 40: 1-9. https://doi.org/10.1016/S0928-8244(03)00309-2

Dar D, Shamir M, Mellin JR, Koutero M, Stern-ginossar N, Cossart P, Sorek R (2016). Term-seq reveals abundant ribo-regulation of antibiotics resistance in bacteria. Science. 80: 352. https://doi.org/10.1126/science.aad9822

Davey ME, Toole GAO (2000). Microbial Biofilms: from Ecology to Molecular Genetics. Microbiology and Molecular Biology Reveiws. 64: 847-867.

Dirita VJ, Engleberg NC, Heath A, Miller A, Crawford JA, Yu R (2000). Virulence gene regulation inside and outside. Philosophical Transactions of the Royal Society of London. 355: 657-665.

Dirita VJ, Mekalanos JJ (1989). Genetic regulation of bacterial virulence. Annual Review of Genetics. 23: 45582.

Dubey AK, Baker CS, Suzuki K, Jones AD, Pandit P, Romeo T, Babitzke P (2003). CsrA Regulates Translation of the Escherichia coli Carbon Starvation Gene, cstA, by Blocking Ribosome Access to the cstA Transcript. Journal of Bacteriology. 185: 4450-4460. https://doi.org/10.1128/JB.185.15.4450

Eberl L (2006). Quorum sensing in the genus Burkholderia. International Journal of Medical Microbiology. 296: 103-110. https://doi.org/10.1016/j.ijmm.2006.01.035

Goebel W, Hacker J (1989). Coordinate regulation of bacterial virulence genes. Perspective Antiinfective Therapy. 2: 99-106. https://doi.org/10.1007/978-3-322-86064-4 14

Gordon CP, Williams P, Chan WC (2013). Attenuating Staphylococcus aureus Virulence Gene Regulation: A Medicinal Chemistry Perspective. Journal of Medicinal Chemistry. 56: 1389-1404. https://doi.org/10.1021/jm3014635

Gottesman S, Storz G (2011). Bacterial Small RNA Regulators: Versatile Roles and Rapidly Evolving Variations. Cold Spring Harbor Perspectives in Biology. 3: 1-16.

Grant AJ, Farris M, Alefounder P, Williams PH, Woodward MJ, Connor CDO (2003). Co-ordination of pathogenicity island expression by the BipA GTPase in enteropathogenic Escherichia coli (EPEC ). Molecular Microbiology. 48: 507-521.

Guijarro JA, Cascales D, García-torrico AI, García-domínguez M (2015). Temperature-dependent expression of virulence genes in fish-pathogenic bacteria. Frontiers in Microbiology. 6: 1-11. https://doi.org/10.3389/fmicb.2015.00700

Ishihama A (2010). Prokaryotic genome regulation: multifactor promoters, multitarget regulators and hierarchic networks. FEMS Microbiology Reviews. 34: 628-645. https://doi.org/10.1111/j.1574-6976.2010.00227.x

Jackson DW, Suzuki K, Oakford L, Simecka JW, Hart ME, Romeo T (2002). Biofilm Formation and Dispersal under the influence of the Global Regulator CsrA of Escherichia coli. Journal of Bacteriology. 184: 290301. https://doi.org/10.1128/JB.184.1.290

Janga SC, Salgado H, Mart1 A, Collado-vides J (2007). Coordination logic of the sensing machinery in the transcriptional regulatory network of Escherichia coli. Nucleic Acids Research. 35: 6963-6972. https://doi.org/10.1093/nar/gkm743

Kerrinnes T, Zelas ZB, Streckel W, Faber F, Tietze E, Tsch H, Yaron S (2009). CsrA and CsrB are required for the post-transcriptional control of the virulence-associated effector protein AvrA of Salmonella enterica. International Journal of Medical Microbiology. 299: 333-341. https://doi.org/10.1016/j.ijmm.2008.09.001

Kitamoto S, Nagao-kitamoto H, Kuffa P, Kamada N (2016). Regulation of virulence: the rise and fall of gastrointestinal pathogens. Journal of Gastroenterology. 51: 195-205. https://doi.org/10.1007/s00535-0151141-5

Kovacikova G, Skorupski K (2002). Regulation of virulence gene expression in Vibrio cholerae by quorum 
sensing: HapR functions at the aphA promoter. Molecular Microbiology. 46: 1135-1147.

Kröger C, Kary SC, Schauer K, Cameron ADS (2017). Genetic Regulation of Virulence and Antibiotic Resistance in Acinetobacter baumannii. Genes. 8: 1-19. https://doi.org/10.3390/genes8010012

Lee K, Periasamy S, Mukherjee M, Xie C, Kjelleberg S, Rice SA (2013). Biofilm development and enhanced stress resistance of a model, mixed-species community biofilm. International Society for Microbial Ecology Journal. 8: 894-907. https://doi.org/10.1038/ismej.2013.194

Linares JF, Moreno R, Fajardo A, Martínez-solano L, Escalante R, Rojo F, Martínez JL (2010). The global regulator Crc modulates metabolism, susceptibility to antibiotics and virulence in Pseudomonas aeruginosa. Environmental Microbiology. 12: 3196-3212. https://doi.org/10.1111/j.1462-2920.2010.02292.x

Liu Q, Yeo W, Bae T (2016). The SaeRS Two-Component System of Staphylococcus aureus. Genes. 7: 1-20. https://doi.org/10.3390/genes7100081

Martı'nez-Antonio A, Collado-vides J (2003). Identifying global regulators in transcriptional regulatory networks in bacteria. Current Opinion in Microbiology. 6: 482-489. https://doi.org/10.1016/j.mib.2003.09.002

Mellies JAYL, Lorenzen E (2014). Enterohemorrhagic Escherichia coli Virulence Gene Regulation. Microbiology Spectrum. 2: 1-17. https://doi.org/10.1128/microbiolspec.EHEC-0004-2013.Correspondence

Miller MB, Skorupski K, Lenz DH, Taylor RK, Bassler BL (2002). Parallel Quorum Sensing Systems Converge to Regulate Virulence in Vibrio cholerae. Cell. 110: 303-314.

Mohanty BK, Kushner SR (2006). The majority of Escherichia coli mRNAs undergo post-transcriptional modification in exponentially growing cells. Nucleic Acids Research. 34: 5695-5704. https://doi.org/10.1093/nar/gkl684

Moreno R, Rosa R La, Yuste L, Madhushani A, Shingler V, Rojo F (2014). The Crc and Hfq proteins of Pseudomonas putida co-operate in catabolite repression and formation of RNA complexes with specific target motifs 1. Environmental Microbiology. https://doi.org/10.1111/1462-2920.12499

Neidig A, Yeung ATY, Rosay T, Tettmann B, Strempel N, Rueger M, Lesouhaitier O, Overhage J (2013). TypA is involved in virulence, antimicrobial resistance and biofilm formation in Pseudomonas aeruginosa. $B M C$ Microbiology. 13: 1-10.

Oliva G, Sahr T, Buchrieser C (2015). Small RNAs , 5' UTR elements and RNA-binding proteins in intracellular bacteria: impact on metabolism and virulence. FEMS Microbiology. 39: 331-349. https://doi.org/10.1093/femsre/fuv022

Peggy AC, Victor JD (2000). Bacterial virulence gene regulation: an evolutionary perspective. Annual Review of Microbiology. 54: 519-565.

Pérez-rueda E, Collado-vides J (2000). The repertoire of DNA-binding transcriptional regulators in Escherichia coli K-12. Nucleic Acids Research. 28: 1838-1847.

Reales-calderón JA, Corona F, Monteoliva L, Gil C, Luis J (2015). Quantitative proteomics unravels that the post-transcriptional regulator $\mathrm{Crc}$ modulates the generation of vesicles and secreted virulence determinants of Pseudomonas aeruginosa. Journal of Proteomics. 4: 450-453. https://doi.org/10.1016/j.dib.2015.07.002

Reitzer L (2003). Nitrogen Assimilation and Global Regulation in Escherichia coli. Annual Review of Microbiology. 57: 155-176. https://doi.org/10.1146/annurev.micro.57.030502.090820

Romeo T, Vakulskas CA, Babitzke P (2013). Post-transcriptional regulation on a global scale: form and function of $\mathrm{Csr} / \mathrm{Rsm}$ systems. Environmental Microbiology. 15: 313-324. https://doi.org/10.1111/j.14622920.2012.02794.x

Rui S, Tse-Dinh YC (2003). Topoisomerase function during bacterial responses to environmental challenge. Frontiers in Bioscience. 8: 256-263.

Sauer K, Camper AK, Ehrlich GD, Costerton JW, Davies DG (2002). Pseudomonas aeruginosa Displays Multiple Phenotypes during Development as a Biofilm. Journal of Bacteriology. 184: 1140-1154. https://doi.org/10.1128/JB.184.4.1140

Schroeder M, Brooks BD, Brooks AE (2017). The Complex Relationship between Virulence and Antibiotic Resistance. Genes. 39:1-23. https://doi.org/10.3390/genes8010039

Sousa SA, Feliciano JR, Pita T, Guerreiro SI, Leitão JH (2017). Burkholderia cepacia Complex Regulation of Virulence Gene Expression: A Review. Genes. 8: 1-15. https://doi.org/10.3390/genes8010043

Steimer L, Klostermeier D (2012). RNA helicases in infection and disease RNA helicases in infection and disease. RNA Biology. 9: 6. https://doi.org/10.4161/rna.20090

Steinmann R, Dersch P (2013). Thermosensing to adjust bacterial virulence in a fluctuating enviroment. Future Microbiology. 8: 85-105.

Stewart PS, Costerton JW (2001). Antibiotic resistance of bacteria in biofilms. Lancet. 358: 135-138.

Storz G, Altuvia S, Wassarman KM (2005). An abundance of RNA Regulators. Annual Review of Biochemistry. 74: 199-217. https://doi.org/10.1146/annurev.biochem.74.082803.133136

Thomas MS, Wigneshweraraj S (2014). Regulation of virulence gene expression. Virulence. 5: 832-835. 
Vakulskas CA, Potts AH, Babitzke P, Ahmer BMM, Romeo T (2015). Regulation of Bacterial Virulence by Csr ( Rsm ) Systems. Microbiology and Molecular Biology Reveiws. 79: $193-224$. https://doi.org/10.1128/MMBR.00052-14

Wassenaar TM, Gaastra W (2001). Bacterial virulence: can we draw the line? FEMS Microbiology Letters. 201: $1-7$.

Wei G, Liu D, Liang C (2004). Charting gene regulatory networks: strategies, challenges and perspectives. Biochemical Journal. 381: 1-12.

Wilson JW, Schurr MJ, Leblanc CL, Ramamurthy R, Buchanan KL, Nickerson CA (2002). Mechanisms of bacterial pathogenicity. Post graduate Medical Journal. 78: 216-224.

Winzer K, Williams P (2001). Quorum sensing and the regulation of virulence gene expression in pathogenic bacteria. International Journal of Medical Microbiology. 143: 131-143.

Zhu J, Miller MB, Vance RE, Dziejman M, Bassler BL, Mekalanos JJ (2002). Quorum-sensing regulators control virulence gene expression in Vibrio cholerae. Proceedings of the National Academy of Sciences. 99: 3129-3134. 